\title{
Smart Urban Design Space
}

\author{
Philipp Skowron \\ Leipzig University, Germany \\ Email: skowron@wifa.uni- \\ leipzig.de \\ Marvin Hubl \\ University of Hohenheim, \\ Germany \\ Email: marvin.hubl@uni- \\ hohenheim.de
}

\author{
Michael Aleithe \\ Leipzig University, Germany \\ Email: aleithe@wifa.uni-leipzig.de
}

\author{
Julian Fietkau \\ Universität der Bundeswehr \\ München, Germany \\ Email: julian.fietkau@unibw.de
}

\author{
Susanne Wallrafen \\ Sozial-Holding der Stadt \\ Mönchengladbach $\mathrm{GmbH}$, \\ Germany \\ Email: s.wallrafen@sozial- \\ holding.de
}

\author{
Bogdan Franczyk \\ Wroclaw University of Economics, \\ Poland \\ Email: \\ bogdan.franczyk@ue.wroc.pl
}

\begin{abstract}
The irreversible process of demographic change, especially in Germany, leads to numerous challenges. According to this, research has to face the task to integrate the constantly ageing population into the urban and public space in such a way that there are as few barriers as possible. With the support of digitalization, so-called smart urban objects are being designed in order to do make integration, so that people and the available technology can be used most efficiently. A special ontology has been developed to meet this demand.
\end{abstract}

\section{INTRODUCTION}

$\mathrm{T}$ he demographic change of a permanently ageing population has become a globally visible phenomenon. Particularly in Germany, the population will be considerably older in the future than it is at present. According to [1], every third person will be older than 65 years of age by 2060 . Corresponding with the tendency of a permanently ageing population goes the fact of changing needs and in daily life. In the era of the inevitable digitalization and in particular the Internet of Things (IoT), the challenge is to what extent digitalization can improve daily life for these ageing population. Accordingly, the concept of providing the urban space with so-called smart urban objects (SUOs) [2] is being pursued to increase the participation of elderly people by digitalization. These SUOs are elements of the urban environment, e.g. lights, information boards and benches, which are connected to a digital information space and allow for implicit or explicit interaction. The desired goal is to increase the feeling of security on urban environment by personalization of these objects. Some of these SUOs are described in detail in [2], [3], [4] , [5] and [6]. The focus of this research is the intersection between the behavior of elderly people, currently referred to as Ambient Assisted Living (AAL), and Smart City. The final focus of this paper is to provide an ontology for classifying these SUOs so that both the technical aspects as well as the aspects of the AAL are considered.

\section{Motivation and Research Question}

The increase in barrier-free accessibility, especially for older persons, will be achieved with the support of SUOs. In order to enable a categorization of these objects, an ontology is required which takes both technical aspects and the view of public health and AAL into account. Based on this kind of ontology, designers of SUOs can consider all aspects mentioned to achieve maximum efficiency of these objects in the later context. In order to sufficiently answer this motivation, following research question is posed, which is the central issue of this article.

How does a taxonomy for the design of SUOs have to be constructed in order to sufficiently consider aspects of Public Health and AAL as well as the technical perspective, so that a maximum increase of barrier-free accessibility is already addressed during the design process?

\section{ReLATED Work}

At this point, approaches and solutions are described and analyzed in terms of the way they answer the research question of this article. Basically, ontologies exist on the one hand in the field of Smart City and on the other hand in the field of so-called Public Health. At this point, both directions will be analyzed in depth and compared with each other, though the research question here characterizes exactly the intersection between these two directions.

In [7] an ontology in the area of Public Health is described, which characterizes in particular the direct situation in the hospital. Here so-called medical classes and medical activations exists. The former include specific diseases, symptoms, therapies, roles and departments in the hospital. The activations subsequently serve to bring these medical classes together in a meaningful relationship and thus describe the applications in the field of Public Health. An ontology-based approach in public health with the support of a geographic information system (GIS) is discussed in [8]. 
The ontology is used for the fusion of data from social and health related issues. Nevertheless, the GIS is the primary focus of the description, and ontology is only used as a tool. So therefore is no further discussion of it. In the contribution of [9] a set of different ontologies is presented, which should support designers in the development of so called AAL and those services. In detail, actors, spaces and devices are modeled and linked so that concrete AAL-elements can be described that have been used within the present study. Overall, this type of modeling is very complex and still has no generic character, meaning that any further use is crucial. A framework for managing the current state as well as the users profile information extracted from the internet and the mobile context is illustrated in [10]. This so called Next Generation Network (NGN) is an ontology for modeling typical users of AAL-services. But these services are only user centric and have no relation to technical issues. Also the platform in [11] offers assistance in communication and information acquisition by providing personalized and context-awared AAL-services. Therefore an ontology is used whre users are the central aspect of the platform. Furthermore this ontology enables a historical view of the users changing characteristics and environment. In view of this explanation, only the user behavior is addressed without encompassing the technical factors. Also in [12] an ontology for structuring daily living activities of users is depicted, whereby a stronger focus is placed on the underlying aspect of AAL and thus on elderly persons. In contrast, the ontology in [13] discusses the technical aspects in terms of best practice for building automation devices and functions and how these underlying models are structured especially in the area of AAL. But in this case there is only a technical view without inclusion of users perspective.

In contrast to solutions of Public Health and AAL, there are some approaches from the Smart City context. These are presented in the following. This Smart City context is characterized by data collected from various distributed systems. Purposing these task in [14] the so called Semantic Web is used for designing a new Smart City ontology. The primary focus is to address the interoperability among the different systems and frameworks for describing Smart City objects. In [15] is an analysis about the impact of Smart City applications observed in the field of energy and transport. Besides [15] describes [16] an ontology to describe the entire Smart City domain. In [17] this description is extended for IoTbased applications. Nevertheless, [14], [15], [16] and [17] all have a strong technical focus and do not mind the user-centered perspective.

In addition to the number of ontologies mentioned so far, a so-called Design Space is described in [18] which enables the characterization and categorization of UI-based elements in the development of applications. This idea would require continuous expansion to include the sensors and applications of the IoT arising.

In summary, a wide spectrum of previous ontologies were presented. These addresses on the one hand the areas of
Public Health and AAL and on the other hand the topic of Smart City. The former ontologies have a strong user-centric focus and the latter are technically very pronounced. However, there is no solution among all approaches that represents a sufficient mix to satisfy the related research question of this article. In addition, the investigated solutions indicate that the aspect of interconnecting the underlying data structure is becoming increasingly important. As a result, this aspect would also have to be integrated more into the ontologies used in this context.

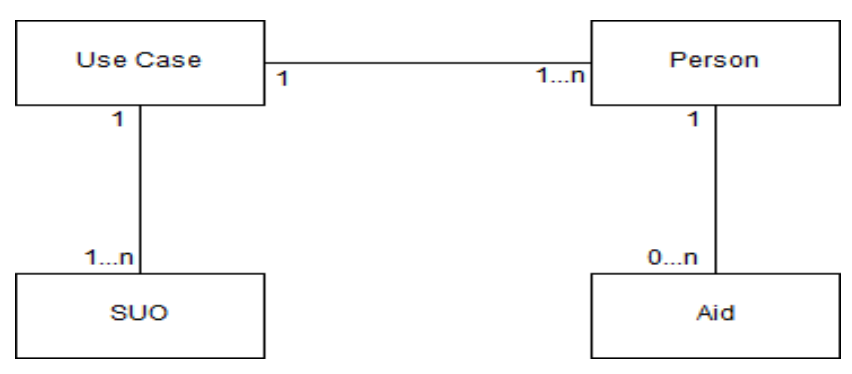

Fig 1. This picture illustrates the entity relationship diagram of the basic relation between smart urban objects (SUOs), the appropriate use case, involved persons and the personalized purpose (aid).

\section{Description of Smart Urban Design Space}

This chapter introduces the so-called Smart Urban Design Space (SUDS). Such a taxonomy meets the above-mentioned full range of criteria in terms of technical aspects, AAL and Public Health. A fundamental idea of this SUDS is the networking of the separate criteria. The basic context is represented graphically in Fig. 1, whereby each use case can be supported by at least one or more SUOs, which are used by at least one or more persons. In order for the SUOs to be used by the persons per use case, it may be necessary to provide additional assistance, which is continuously referred to as aid.

Against this background, a use case is the concrete scenario in which the elderly person(s) can use the digital support outdoors (outside buildings). Concrete examples in this context are an adaptive lighting system of the area to be walked in during a walkway, an adaptive park bench, which adapts to the individual sitting height of the respective person as well as intelligent information spotlights, which provide personalized information of the urban space to be visited. These examples are presented in detail in chapter 5 .

Within the SUDS, the three criteria SUO, Aid, and Person exist for each use case, with their corresponding subordinate properties. In this regard, an overview of the entire taxonomy is shown in Fig. 2. A person has so-called competencies, which are continuously referred to as skills. These include speaking, seeing, hearing, cognitive skills such as easy logical thinking and movement, which in this case refers to walking without aids. The SUO contains the five criteria $a c$ tuator, sensor, parallelization, personalization and interaction sensor. The interaction sensor describes which human 
sense for an interaction of the SUO is required. It distinguishes between seeing, hearing and haptic handling such as using a touch pad. In addition to operating sensors, there is also the criterion of technical sensors, which is referred to merely as sensors within this taxonomy. There are mechanical, piezoelectric, capacitive, inductive, optical, magnetic and signal-based practices. The latter symbolize the provision of information by an external information source. Similar to technical sensor technology, the actuator also distinguishes between mechanical, signal-based, optical, thermal and acoustic variants. Personalization classifies the SUO according to whether each individual person is addressed individually, whether a group of people is addressed (cluster) or whether no individual personalization (general) is satisfied. In this context, there is also the criterion of parallelization, whether the SUO differentiates only single-user or multiuser in the respective use case. Similar to the SUO, the aid has a shortened set of criteria. The interaction sensor, actuator and sensor are used, with the latter describing the technical perspective. The characteristics of these criteria are analogous to those of the SUO.

\section{Case Study "UrbanLife+"}

In the research project UrbanLife+, the autonomy and participation of senior citizens in urban areas is explored in such a way that they can be increased. For this purpose, urban objects in Mönchengladbach are to be transformed into SUOs with the help of innovative human-technology interaction approaches, which provide senior citizens with technical support in line with their needs and enable them to move around the city safely [2] [5]. Three use cases are pre- sented for these addressed solutions, which are then classified in the SUDS. These use cases are Adaptive Lighting, Adaptive Park Bench and the Information Radiators. In the following these are explained briefly and the classification in the SUDS is discussed individually. Overall it is represented in Fig. 2.

\section{A. Adaptive Lighting System}

The system of the Adaptive Lighting improves the feeling of safety on elderly people especially in dark areas at night by personalized and position-dependend variation of intensity and/or color of the light [1] [4].

\section{B. Adaptive Park Bench}

Adaptive Park benches are a kind of smart seats, that can adjust to individual anthropometric measures of people. Thereby the usability of the seats is enhance which in turn also enhances safe usage. Particularly older people face severe problems in sitting down and standing up at common seats. The reason is that, the gap between standover and the height of the seat surface imposes trouble when the older people have weakened leg muscles, impaired balance or general difficulties in bending their knees. For this reason the seat surface of the adaptive park bench can lift up to the standover of a pedestrian, which actively supports in sitting down and standing up. For ergonomic sitting the seat surface will be adjusted to the sitting person's popliteal height. More technical details are described in [2] [19].

\section{Information Radiators}

Information Radiators are a class of devices capable of displaying dynamic information while installed at a static

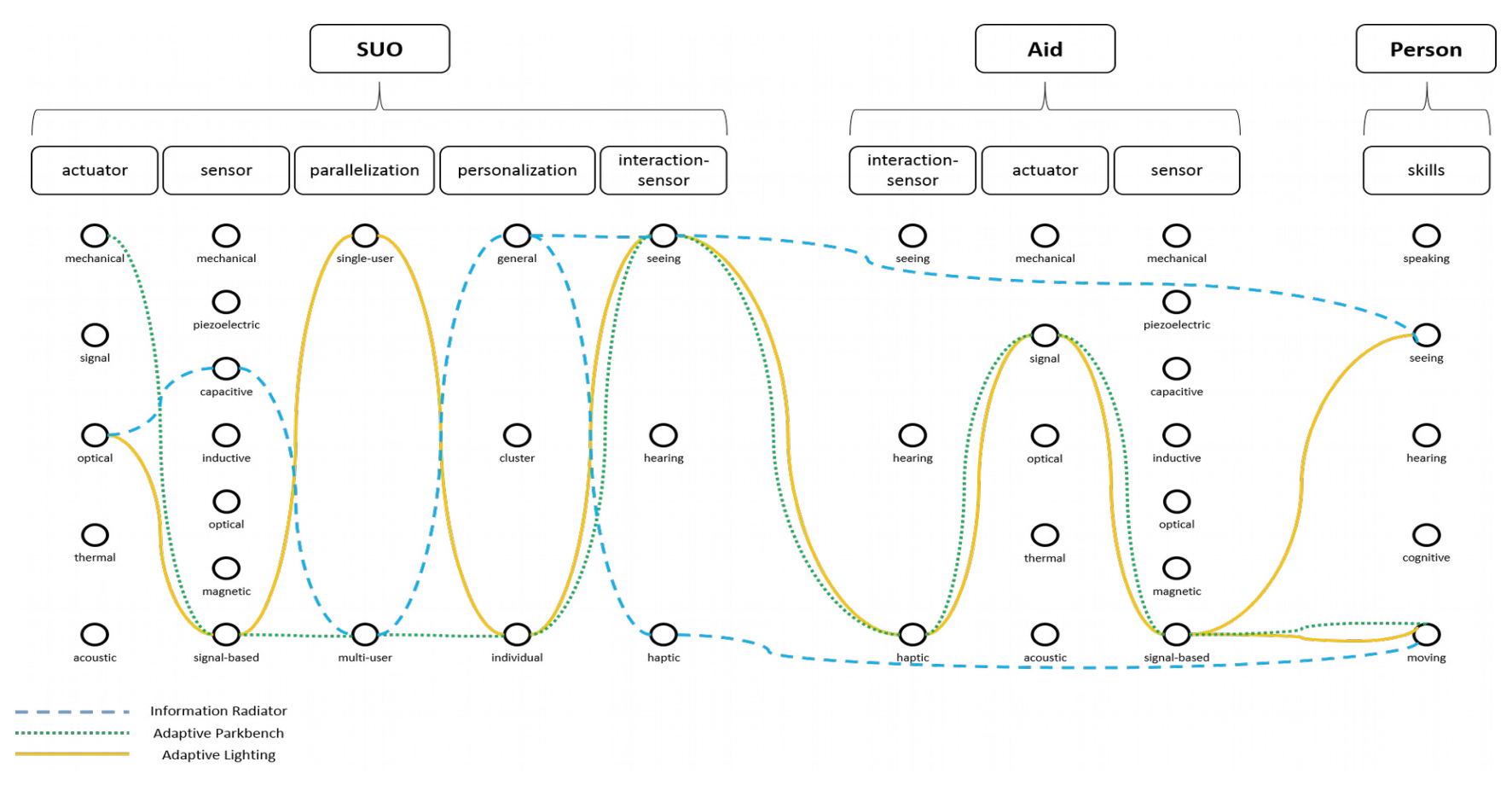

Fig 2. This figure is the representation of the so-called Smart Urban Design Space (SUDS). It shows the basic categories SUO, Aid and Person, with the available properties. By combining the properties of these categories addressed by the corresponding use case, a visualization similar to a dendogram is generated. Furthermore in this figure, the three use cases are arranged in the SUDS. These use cases include the Adaptive Lighting, the Adaptive Park Bench and the Information Radiator, each of which is marked in the legend. 
position in a public or semi-public environment. They can range from large interactive screens which can be used via touch, to small low-power devices equipped with low-resolution LED displays. The informational content they show is related to the local context. This includes, but is not limited to, offerings by commercial and noncommercial actors in the vicinity (such as stores, restaurants, cinemas, community centers, sports clubs, etc). The concept is discussed in more detail in [20].

\section{Discussion}

As a result of the taxonomy of the SUDS developed in this paper, a rather interdisciplinary classification of the socalled SUOs has been successfully achieved, without getting stuck in technical details in this context, nor without too one-sided a view of social criteria affecting the user. In this context, the previous SUOs were specifically classified in the SUDS (see Figure 3). The SUOs classified so far include Adaptive Lighting, the Adaptive Park Bench and the Information Radiators. In addition to these existing SUOs, there is also the possibility of continuously classifying new ones in order to visualize the essential aspects of the local field of knowledge. The classification in this taxonomy (Figure 3) shows that more or less all SUOs have similar characteristics regarding their categories. For example, in relation to the interaction sensor, which is only haptically or optically pronounced in all previous objects. Consequently, an essential motivation for further SUOs would be to include acoustic signals in order to increase the intersection between the technical and personal skills

\section{Conclusion and Outlook}

Concerning the research question within this article, a taxonomy called SUDS was constructed which merges the required aspects of AAL, Public Health and technical aspects and makes them usable for integrating so-called SUOs.

In the future, potentially beneficial SUOs could be determined and designed with the support of the SUDS, which do justice to the aspects of AAL and Public Health without violating the technical conditions.

\section{ACKNOWLEDGEMENT}

This work was fully conducted in the scope of the research project UrbanLife+ (16SV7442), funded by the German Ministry of Education and Research.

\section{REFERENCES}

[1] https://de.statista.com/statistik/daten/studie/71539/umfrage/ bevoelkerung-in-deutschland-nach-altersgruppen/ , last accessed: 2019-03-11

[2] Hubl, M., Skowron, P., Aleithe, M.: Towards a Supportive City with Smart Urban Objects in the Internet of Things: The Case of Adaptive Park Bench and Adaptive Lights. In: Position Papers of the 2018 Federated Conference on Computer Science and Information Systems (FedCSIS). Annals of Computer Science and Information Systems (ACSIS) 16. Maria Ganzha, Leszek A. Maciaszek, Marcin Paprzycki (eds.), pp. 51-58, (2018). doi:10.15439/2018F118
[3] Kötteritzsch, A., Koch, M., Wallrafen, S.: Expand Your Comfort Zone! Smart Urban Objects to Promote Safety in Public Spaces for Older Adults. In: Adjunct Proceedings of UbiComp 2016, ACM Press, (2018) doi:10.1145/2968219.2968418

[4] Aleithe, M., Skowron, P., Franczyk, B., Sommer, B.: Data modeling of smart urban object networks. In: Proceedings of the International Conference on Web Intelligence (WI '17), pp. 1104-1109 (2017). doi:10.1145/3106426.3117759

[5] Aleithe, M., Skowron, P., Schöne, E., Franczyk, B.: Adaptive Lighting System as a Smart Urban Object. In: Communication Papers of the 2018 Federated Conference on Computer Science and Information Systems (FedCSIS 2018). Annals of Computer Science and Information Systems (ACSIS) 17, Maria Ganzha, Leszek A. Maciaszek, Marcin Paprzycki (eds.), pp. 145-149, (2018). doi:10.15439/2018F30

[6] https://www.urbanlifeplus.de/, last accessed: 2019-02-26

[7] Abinaya, Kumar, V., Swathika: Ontology Based Public Healthcare System in Internet of Things (IoT). In: Procedia Computer Science 50, pp. 99-102, (2015). doi:10.1016/j.procs.2015.04.067

[8] Gür, N., Sanchez, L. D., Kauppinen, T.: GI Systems for Public Health with an Ontology Based Approach. In: Proceedings of the AGILE'2012 International Conference on Geographic Information Science, Avignon, pp. 86-91 (2012). ISBN: 978-90-816960-0-5

[9] Mocholi, J. B., Sala, P., Fernandez-Llatas, C., Naranjo, J. C.: Ontology for Modeling Interaction in Ambient Assisted Living Environments. In: XII Mediterranean Conference on Medical and Biological Engineering and Computing (MEDICON 2010), pp. 655658 (2010). doi:10.1007/978-3-642-13039-7_165

[10] Moreno, P. A., Hernando, M. E., Gomez, E. J.: AALUMO: A User Model Ontology for Ambient Assisted Living Services Supported in Next-Generation Networks. In: XIII Mediterranean Conference on Medical and Biological Engineering and Computing 2013, pp. 12171220 (2013). doi:https://doi.org/10.1007/978-3-319-00846-2_301

[11] Fredrich, C., Kuijs, H., Reich, C.: An Ontology for User Profile Modeling in the Field of Ambient Assisted Living. In: SERVICE COMPUTATION 2014 : The Sixth International Conferences on Advanced Service Computing. (2014). ISBN: 978-1-61208-337-7

[12] Woznowski, P. R., Tonkin, E. L., Flach, P. A.: Activities of Daily Living Ontology for Ubiquitous Systems: Development and Evaluation. In: Sensors 2018, 18, 2361. (2018) doi: $10.3390 / \mathrm{s} 18072361$

[13] Butzin, B., Golatowski, F., Timmermann, D.: A survey on information modeling and ontologies in building automation. In: Conference: IECON 2017 - 43rd Annual Conference of the IEEE Industrial Electronics Society. (2017). doi:10.1109/IECON.2017.8217514

[14] Abid, T., zarzour, H., Laouar, M. r., Khadir, M. T.: Towards a smart city ontology. In: Conference: 2016 IEEE/ACS 13th International Conference of Computer Systems and Applications (AICCSA). (2016). doi:10.1109/AICCSA.2016.7945823

[15] Komninos, N., Bratsas, C., Kakderi, C., Tsarchopoulos, P.: Smart city ontologies: Imroving the effectivenessof smart city applications: In: Journal of Smart Cities 1(1), pp. 1-16 . (2015). doi:10.18063/JSC.2015.01.001

[16] Ramaprasad, A., Sanchez-Ortiz, A., Syn, T.:A Unified Definition of a Smart City. In: EGOV 2017, LNCS 10428, pp. 13-24, (2017). doi:10.1007/978-3-319-64677-0 2

[17] Gyrard, A., Zimmermann, A., Sheth, A.: Building IoT-Based Applications for Smart Cities: How Can Ontology catalogs Help? In: IEEE Internet of Things Journal 5(5), pp. 3978-3990. (2018). doi:10.1109/JIOT.2018.2854278

[18] Minon, R., Paterno, F., Arrue, M.: An Environment for Designing and Sharing Adaption Rules for Accessible Applications. In: EICS'13. (2013)

[19] Hubl, M.: Adaption rule for simultaneous use of smart urban objects from a fairness perspective. In: Proceedings of the 20th IEEE International Conference on Business Informatics (CBI 2018), pp. 89-98. (2018). doi:10.1109/CBI.2018.00019

[20] Koch, M., Kötteritzsch, A., Fietkau, J.: Information radiators: using large screens and small devices to support awareness in urban space. In: Proceedings of the International Conference on Web Intelligence (WI '17), pp. 1080-1084 (2017). doi:10.1145/3106426.3109039 